(2) Open Access Full Text Article

\title{
An Open, Uncontrolled Pilot Study on I2-Week Use of VagiVital for Treatment of Vulvovaginal Atrophy in Breast Cancer Patients Undergoing Adjuvant Aromatase-Inhibitor Therapy
}

\author{
Aino Fianu Jonasson' \\ Mikael Åstrom (D) ${ }^{2}$ \\ 'Department of Clinical Sciences, \\ Intervention, and Technology, Division of \\ Obstetrics and Gynaecology, Karolinska \\ Institute, Stockholm, Sweden; ${ }^{2}$ StatCons, \\ Malmö, Sweden
}

Purpose: This pilot study assessed the efficacy of 12 weeks of daily treatment with a vaginal gel based on a water-based cellulose gel in reducing the severity of moderatesevere symptoms of vulvovaginal atrophy (VVA) and followed adverse events in female breast cancer patients undergoing treatment with adjuvant aromatase- inhibitor therapy.

Methods: In this open, uncontrolled pilot study, the efficacy and safety of the gel during a treatment period of 12 weeks (daily $1 \times 1 \mathrm{~mL})$ were evaluated $(\mathrm{n}=28)$. The gel is made of water and hypromellose, a hydropropylmetyl cellulose, which works by coating the vagina, and was developed to treat moderate-severe symptoms of VVA. The primary efficacy variable was the most bothersome symptom (MBS; among vulvovaginal irritation and itching, vaginal dryness, dysuria, or dyspareunia) self-identified at baseline on a four-point scale.

Results: A total of 28 patients fulfilled all entry criteria and had data available after the start of treatment. Treatment with the gel reduced MBS scores from baseline ( $n=28$, mean 2.71) to week $12(\mathrm{n}=27$, mean 1.33, mean reduction 1.37; $p=0)$, and reduced the overall total scores for VVA symptoms from a mean of 5.39 at baseline to 2.25 at week $12(p=0)$. Eleven subjects (39\%) reported 19 AEs.

Conclusion: A 12-week treatment with the gel significantly reduced the severity of MBSs and VVA symptoms, improved quality of life, and was safe to use in women with breast cancer undergoing adjuvant aromatase-inhibitor therapy. In view of the beneficial effects of nonhormonal treatments, for cancer patients in particular, the water-based cellulose gel VagiVital is a suitable candidate for first-choice treatment of VVA symptoms in breast cancer patients and in women predisposed to cancer.

Keywords: vulvovaginal atrophy, MBS, nonhormonal therapy, dyspareunia, vaginal dryness, adjuvant AI therapy, breast cancer

\section{Introduction}

Treatment of breast cancer with systematic therapies, such as chemotherapy and endocrine therapy, causes a variety of symptoms that can impair quality of life (QoL) for many breast cancer patients. Vulvovaginal atrophy (VVA), similar to that developing after menopause as a result of reduced production of estrogen, is
Correspondence: Aino Fianu Jonasson Department of Clinical Science, Intervention and Technology Division of Obstetrics and Gynecology, Karolinska Institute, Stockholm, I7I 77, Sweden Tel +46-70-483-6028

Email aino.fianu.jonasson@ki.se 
a commonly experienced condition in these patients. Reduced estrogen production and decreased levels of circulating estrogen reaching urinary and vaginal receptors lead to decreased fluid production within the vagina, resulting in a less elastic, thinner, and more fragile mucosa. ${ }^{1}$ Normal lactobacilli production is also affected and leads to a higher $\mathrm{pH}$ in the vaginal area. Typical symptoms associated with VVA, such as vaginal dryness, vulvovaginal irritation/itching, dysuria, and dyspareunia, are often accompanied by other painful symptoms, such as stinging, minor lesions, or soreness in the vaginal area and at the vaginal opening, severely impacting QoL. ${ }^{2}$ VVA is also often associated with elevated vaginal $\mathrm{pH}$, increasing susceptibility to infection. ${ }^{3,4}$ In contrast to some other estrogen-related symptoms observed in menopausal women, such as hot flushes, vaginal symptoms generally persist or worsen with age. ${ }^{5,6}$ In women experiencing VVA symptoms, the first-line treatment is currently local intravaginal administration of estrogen. ${ }^{7-9}$ However, in cancer patients, this treatment poses safety concerns relating to systemic absorption of vaginal estrogen. $^{7-9}$ Vaginal moisturizers are an alternative option for local nonhormonal treatment of VVA. ${ }^{10,11}$ Such moisturizers can help to reduce vaginal $\mathrm{pH}^{12,13}$ without affecting follicle-stimulating hormone or estradiol serum concentrations, making them safe for use in women who must avoid hormonal treatment, such as breast cancer patients. ${ }^{14-16}$ In a previous study ${ }^{18}$ evaluating the efficacy of a vaginal gel moisturizer based on aqueous hypromellose gel in reducing the severity of various VVA symptoms in menopausal women, we demonstrated a statistically significant reduction in clinical symptoms after 12 weeks of treatment, with similar magnitude of reduction as previously reported for estrogen-based products. ${ }^{17}$

In the present pilot study, we investigated the ability of a water based cellulose gel to reduce the severity of moderate-severe VVA symptoms in women with breast cancer undergoing treatment with adjuvant aromataseinhibitor therapy. The women undergoing treatment with aromatase inhibitors were contraindicated for local estrogen therapy ${ }^{19}$ and thus needed nonestrogen products. This was the reason for developing this water-hypromellose product. VVA is associated with increased $\mathrm{pH}$, which creates an environment more susceptible to infections.

The subjects selected for this study were assessed as being in stable condition, as defined by inclusion and exclusion criteria, and thus it was assumed that no change would occur over time if the treatment had no effect. ${ }^{20-23}$ As subjects were considered stable over time, a change in VVA symptoms was considered a result of using the gel. However, the results should be considered with caution, given the absence of a control group.

\section{Methods}

All work performed in the study was in compliance with International Council for Harmonisation Guideline for (CPMP/ICH/135/95). It was also in compliance with ISO 14155:2011 and with relevant regional regulations (ie, European Directive 2001/20/EC), and the ethical guidelines of the World Medical Association. The clinical protocol for the study was approved by the Swedish Ethical Review Authority (2019-02346), and all participants voluntarily provided signed informed consent. The clinical work was carried out at the Department of Gynaecology and Reproductive Medicine, Karolinska University Hospital, Stockholm, Sweden from June 2019 to June 2020 (first patient included on June 14, 2019; last patient final visit on June 24, 2020). Breast cancer patients with VVA symptoms were recruited from patients treated at the department. Four visits per subject were included: a screening (baseline) visit (visit 1), treatment follow-up in after 4 weeks (visit 2), an end-of-treatment visit after 12 weeks (visit 3), and a telephone follow-up after 14 weeks (visit 4). The product evaluated was VagiVital gel, which is manufactured by Peptonic Medical and was delivered to each patient in a polythene bag, together with an applicator and a leaflet in Swedish with instructions for use. Initial training was provided by clinic staff, and then each patient self-administered an initial dose $(1 \mathrm{~mL})$ of the gel at the clinic. All patients were instructed to apply a further $1 \mathrm{~mL}$ of the water-based cellulose gel once daily at around the same time of day for 12 weeks. The $\mathrm{pH}$ of the product has been adjusted to 3.75 to diminish the higher $\mathrm{pH}$ associated with VVA. The product's osmolarity is $45-80 \mathrm{mOsmol} / \mathrm{kg}$. Osmolarity in the vagina is approximately 280-300 $\mathrm{mOsmol} / \mathrm{kg}$, ie, hypoosmolarity exists and results in water transfer/passes into the mucosa of the vagina.

\section{Study Design}

This open, uncontrolled pilot study was performed to evaluate the effects of the gel in breast cancer patients with VVA symptoms over a 12-week treatment period and a 2-week follow-up period. The primary objective was to assess its efficacy in improving the most bothersome symptoms (MBSs) of VVA from baseline until week 12 
(change until week 4 was a secondary objective) in women undergoing treatment for breast cancer with adjuvant aromatase-inhibitor therapy. The severity of MBSs was identified by each patient at baseline. Safety objectives were to evaluate the type and frequency of adverse events (AEs), serious AEs, adverse device effects, serious adverse device effects, and device deficiencies arising during the treatment period. Secondary efficacy end points evaluated included changes (from baseline until week 4 and 12) in vaginal $\mathrm{pH}$, severity of other VVA symptoms, and QoL parameters (week 12 only). Following initial screening and baseline assessments, all participants were shown how to self-administer the intravaginal gel (1 mL once daily). They were asked to continue daily self-administration for 12 weeks using the applicator provided at approximately the same time every day as the initial dose. Clinical evaluations were made at visit 1 (baseline): week 0 , day 0 ; visit 2: week 4, day $28 \pm 3$; visit 3 : week 12 , day $84 \pm 5$; and visit 4: week 14, day $98 \pm 3$.

\section{Study Population}

Females aged 18 years or older, diagnosed with breast cancer, currently receiving adjuvant aromatase-inhibitor therapy, and reporting vulvovaginal atrophy symptoms (vaginal dryness, vulvovaginal irritation/itching, pain when urinating, dyspareunia, vaginal bleeding associated with sexual activity), but otherwise in good health and who provided signed informed consent were considered eligible for participation in the study. The women were asked to abstain from sexual activity and vaginal douching for 24 hours prior to vaginal $\mathrm{pH}$ measurements conducted at screening (visit 1) and visits 2 and 3. Women reporting contraindications to water-based cellulose-gel therapy and/ or any allergy to components of the applicator device were excluded from participating in the study, as were women with a history of drug and/or alcohol abuse within the year leading up to the study. Participating women were requested not to use any prescription or over-the-counter medication, including phytoestrogens and herbal medicinal products, or any hormonal intrauterine devices with known estrogen/progestin effects for 12 weeks prior to screening. They were also asked not to use any type of vaginal lubricant or moisturizer in the 24 hours prior to screening. Further, they were asked not to use various products containing estrogen alone or estrogen/progestin for specific periods: vaginal hormonal products (gels, rings, creams, suppositories, pellets/injectables) for 12 weeks prior to screening, transdermal estrogen or estrogen/progestin products, including percutaneous estrogen gels, for at least 12 weeks prior to screening, oral estrogen and/or progestin therapy in the 12 weeks prior to screening, intrauterine progestin therapy in the 12 weeks prior to screening, progestin implants and estrogen-alone injectable drug therapy in the 12 weeks prior to screening, and (f) estrogen pellet therapy or pregestational injectable drug therapy in the 6 months prior to screening. Women with contraindications to any planned study procedure were excluded from the study. Those who had participated in another clinical trial within 90 days prior to screening or had received a trial drug within 3 months prior to the initial dose of the gel or were likely to participate in another clinical trial or receive another trial drug during the study were also excluded. In the opinion of the principal investigator, those who could not safely participate in the study or comply with the protocol requirements and women with any other reason for exclusion under the Declaration of Helsinki were not included. A final total of 32 women were included from an initial panel of 40 , and 28 fulfilled the entry criteria and had data postbaseline.

\section{Assessments}

Efficacy was evaluated based on change in severity of MBSs of VVA identified by the patient at baseline and on reported changes in severity of other individual VVA symptoms, measured changes in vaginal $\mathrm{pH}$, and evaluation of QoL parameters from baseline onward. VVA symptoms evaluated on visits 1 (screening/baseline), 2 (week 4) and 3 (week 12) were:

- vaginal dryness (none, mild, moderate, severe)

- vaginal and/or vulval irritation/itching (none, mild, moderate, severe)

- dysuria, ie, pain, burning, or stinging when urinating (none, mild, moderate, severe)

- dyspareunia, ie, vaginal discomfort and/or pain during vaginal sexual activity (none, mild, moderate, or severe)

- vaginal bleeding associated with vaginal sexual activity (yes/no).

The severity of four of these VVA symptoms (vaginal dryness, vaginal and/or vulvar irritation/itching, dysuria, and dyspareunia) was assessed in interviews with the patients and by a self-assessment questionnaire on VVA symptoms and QoL. Participants were asked to complete 
the questionnaire at visit 1 and on visits 2 and 3 using a four-point scale: 0 (none), 1 (mild), 2 (moderate), :3 (severe). In the questionnaire, participants were also asked about absence/presence of vaginal bleeding associated with vaginal sexual activity and whether vaginal discomfort and/or pain was a reason for them not engaging in sexual activity. Vaginal $\mathrm{pH}$ was measured at screening and on visits 2 and 3 using a $\mathrm{pH}$ meter to determine $\mathrm{pH}$ in vaginal smears collected from the lateral vaginal wall according to laboratory instructions. Associated conditions or symptoms were asked about and documented in patients' medical history but were not part of this pilot study.

Safety assessments were performed throughout the study, including reporting of AEs and adverse device effectss. Relevant data were collected and evaluated at visits 2,3 , and 4 (telephone follow-up). Safety was evaluated based on measurements of vital signs for each participant on each visit. These measurements included physical, gynecological and breast examinations, and urine analysis.

\section{Statistical Analysis}

The number of patients included in the study was not based on any statistical calculations. However, based on previous clinical experience, the number of patients included was expected to be large enough to draw valid scientific conclusions. Efficacy of the test product was evaluated based on data from all correctly included patients who used the study medication during the full 12-week treatment period and who had at least one efficacy assessment postbaseline. Baseline measurement was defined the last valid measurement before the first dose of the gel. Missing data were not imputed.

The Wilcoxon signed-rank test was used to analyze changes over time in continuous and ordered categorical data, and binomial tests to analyze changes over time in binary variables. All $p$-values presented in results are based on two-sided tests. Adjustment for multiplicity was not performed. Two-sided $p<5 \%$ was classified as statistically significant, even though adjustment for multiple comparisons were not made, and hence the risk of false-positive findings needs to be taken into consideration when drawing conclusions.

\section{Results}

A total of 32 patients were screened, 29 fulfilled the entry criteria and started treatment, but one patient was withdrawn early and hence no data were available for her following the start of treatment. This meant that 28 patients were included in the statistical analyses. Demographic characteristics and baseline characteristics (age, height, weight, body-mass index, tobacco and alcohol history) for all participants are shown in Table 1.

\section{Primary Efficacy End Point}

\section{Most Bothersome Symptoms of VVA}

For the VVA symptoms identified as MBSs by patients at baseline, the mean baseline value was $2.71(n=28)$ and at week 12 was $1.33(n=27)$. Mean improvement from baseline until week 12 was $1.37(p=0)$, see Figure 1 .

\section{Secondary Efficacy End Point}

For MBSs of VVA at week 4, the mean was $1.81(n=27)$, with mean reduction from baseline of $0.89(p=0$, Figure 1$)$.

$\mathrm{pH}$ decreased statistically significantly from a mean of 7.57 at baseline to a mean of 7.05 at week $12(p=0.0009$, Figure 1).

For vaginal dryness, mean baseline value was 2.64 and had reduced to 1.11 at week $12(p=0)$. Mean at week 4 was 1.56 ( $p=0$, Figure 1).

For dyspareunia (sexually active patients only, nine at baseline, ten at week 4 , and eleven at week 12), statistical significance $(p=0.0313)$ with noted for time when comparing

Table I Demographic characteristics and baseline characteristics of subjects included in the study $(n=28)$

\begin{tabular}{|c|c|c|c|c|}
\hline & Mean & Minimum & Maximum & SD \\
\hline Age, years & 61 & 40 & 82 & 8.90 \\
\hline Height, cm & 167.68 & 160.00 & 182.00 & 5.35 \\
\hline Weight, kg & 68.02 & 53.00 & 82.00 & 7.50 \\
\hline \multirow[t]{2}{*}{ Body-mass index } & 24.20 & 19.00 & 32.03 & 2.61 \\
\hline & $\mathbf{n}$ & \multicolumn{3}{|c|}{$\%$} \\
\hline \multicolumn{5}{|l|}{ Use of tobacco: } \\
\hline Never & 22 & \multicolumn{3}{|c|}{79} \\
\hline Current & 0 & \multicolumn{3}{|c|}{0} \\
\hline Former & 6 & \multicolumn{3}{|c|}{21} \\
\hline \multicolumn{5}{|l|}{ Alcohol consumption: } \\
\hline Never & 3 & \multicolumn{3}{|c|}{11} \\
\hline Current & 25 & \multicolumn{3}{|c|}{89} \\
\hline
\end{tabular}



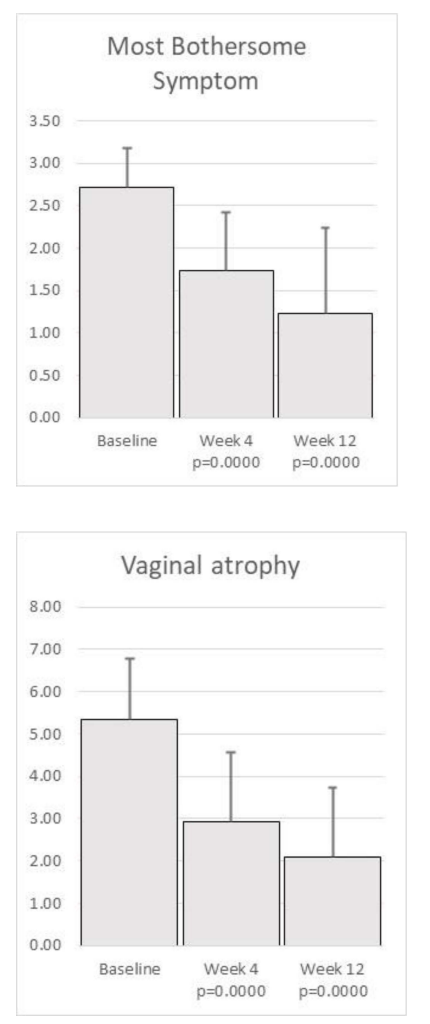
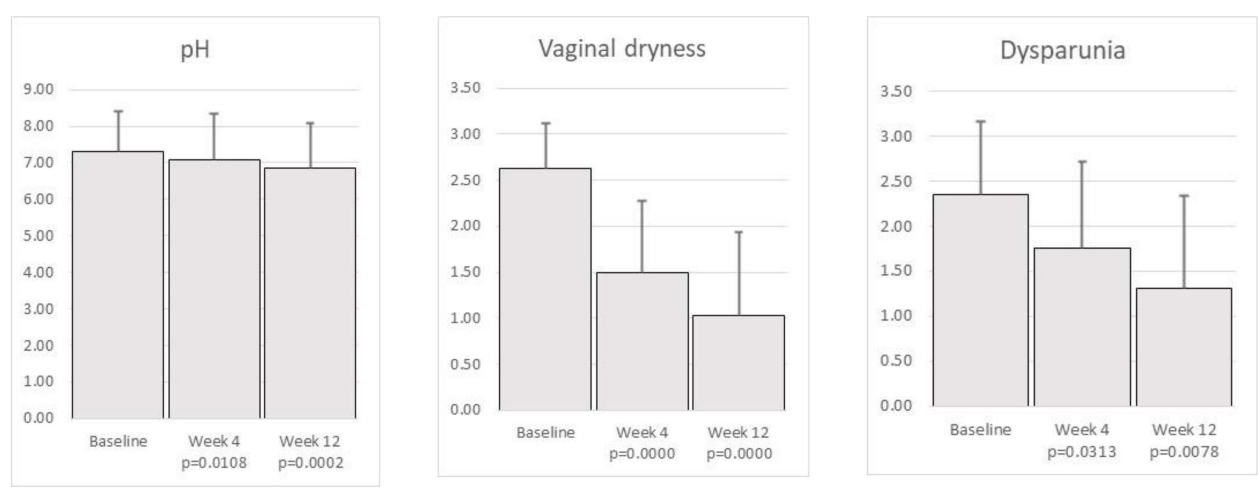

Figure I Means \pm SD for efficacy variables by time of investigation. The $p$-values represent comparison to baseline.

baseline (mean 2.56) and week 12 (mean 1.45) values. At week 4 , the mean was $1.90(\mathrm{p}=0.0625$, Figure 1$)$.

For Vaginal atrophy total score, mean baseline value was 5.39 and had reduced to 2.25 at week $12(p=0)$. At week 4, this was 3.04 ( $p=0$, Figure 1).

For irritation/Itching, a statistically significant ( $p=0.0012)$ improvement was identified from baseline (mean 1.21) to week 12 (mean 0.54). At week 4, the mean was $0.59(p=0.0002$, Figure 1$)$.

For dysuria severity, mean baseline value was 0.71 , at week 40.19 ( $p=0.0024)$, and at week 120.04 ( $p=0.0010$, Figure 1$)$.

In summary, we identified most of the improvement after 4 weeks of treatment, but improvements were even better after 12 weeks.

\section{Other Individual VVA Symptoms and QoL-Evaluation Parameters}

The number of sexually active women had increased from nine $(32 \%)$ at baseline to ten $(37 \%)$ after 4 weeks of treatment and increased further to eleven patients $(39 \%)$ after 12 weeks of treatment (Table 2). The increase was not statistically significant, but it should be noted that statistical power was limited, as the number of nonsexually active women at baseline was 19 .
Evaluation of data collected via the QoL questionnaire showed that subjects experienced statistically significant improvements on QoL parameters from baseline to week 12. Particular improvements were reported for issues addressed by questions 17-22 (parameters evaluating difficulties with urination frequency, urine leakage, and dyspareunia). These symptoms, which strongly affect QoL in VVA patients, had significantly $(p=0.0008)$ improved at week 12 compared with baseline.

\section{Safety}

Eleven of the initial 28 patients (39\%) reported 19 AEs after starting treatment, with common cold/fever being the most frequently reported (five), followed by urinary tract infections

Table 2 Sexual activity status

\begin{tabular}{|l|c|c|c|}
\hline $\begin{array}{l}\text { Vaginal intercourse last 3 } \\
\text { months }\end{array}$ & Baseline & Week 4 & $\begin{array}{c}\text { Week } \\
\text { I2 }\end{array}$ \\
\hline Yes & $9(32 \%)$ & $10(37 \%)$ & II (39\%) \\
No (because of pain) & $15(54 \%)$ & $11(41 \%)$ & II (39\%) \\
No (reason unknown) & $4(14 \%)$ & $6(22 \%)$ & $6(21 \%)$ \\
Total & 28 & 27 & 28 \\
\hline
\end{tabular}

Notes: change in vaginal intercourse since baseline (binomial test): $p=0.5000$ (week 4), $p=0.6250$ (week I2). 
(threes). A majority (eleven) of these AEs were reported to be of moderate severity. No concerns related to safety and tolerability arose based on vital signs, physical examinations, clinical evaluations, gynecological examinations, urine tests, transvaginal ultrasound, scans, or vaginal smears. Of the 19 reported AEs, eight were considered to have a possible relationship with the treatment, while eight considered unlikely to be related and three dismissed as unrelated. By the end of the study, all AEs had been resolved except for two (vitamin $\mathrm{B}_{12}$ deficiency and nerve pain in the back). Both these were considered unrelated or unlikely to be related, to the treatment. No severe AEs were reported during this study.

\section{Discussion}

The results obtained in this study are applicable to a population meeting the entry criteria for the study, specifically women aged 18 years or older diagnosed with breast cancer undergoing current adjuvant aromatase-inhibitor treatment, experiencing moderate-severe VVA symptoms, sexually active or desiring to be so, but abstaining because of dyspareunia, and otherwise in good health. The study population was considered tn stable condition and observed changes over time was considered theresult of using the gel.

For the primary objective (ie, improvement In MBSs of VVA identified by the patient after 12 weeks of treatment), the results showed that the gel had a highly statistically significant positive effect compared with baseline. Highly significant improvements were also observed for the main secondary efficacy en points (vaginal $\mathrm{pH}$, dryness, irritation/itching, dysuria, dyspareunia). Evaluation of QoL parameters using the questionnaire revealed a significant increase in QoL in the study population. A reduction in vaginal bleeding associated with sexual activity was reported, but was not statistically significant, probably due to low statistical power owing to the very low number of patients $(n=4)$ available for this analysis.

One interesting improvement reported by one patient during interviews within the study period was that she no longer needed to get up to urinate at night, whereas before participation in the study she had had to get up twice per night. When the treatment ended, the need to urinate during the night returned. According to the principal investigator, this had been reported by other patients using the gel in earlier studies.

The most commonly reported AEs were common cold/ fever (five) and urinary tract infection (three). In two patients treated for urinary tract infections at screening, the infection returned during the study, in both cases as an asymptomatic infection. Some of the reported AEs were considered likely to have a possible relationship to the treatment. For instance, in our previous study, urinary tract infection was found to be more prevalent, ${ }^{18}$ probably a consequence of the subjects resuming sexual activity.

During the course of the study, the COVID-19 pandemic emerged, which affected the study in terms of difficulty in recruiting patients and resulted in only 29 enrolled patients compared with the planned 40 patients, but still the study shows conclusive results. The main limitation of the study is that it provides no formal proof, since a control group was not included. However, the study population was stable (see aforementioned justification), and the results were in line with previous findings of similar reductions in VVA symptoms in menopausal women treated with water-based cellulosegel estrogen-based products. ${ }^{17,18}$ This confirms the benefits of using the aqueous hypromellose-based vaginal gel as first-choice treatment in a broad patient population experiencing VVA symptoms. Further studies on the efficacy of this water-based cellulose gel in groups of women with other conditions, such as vulvodynia/vestibulodynia, are warranted. Systematic investigation is also needed to confirm or reject the anecdotal evidence that this gel decreased the need for nightly micturition.

During interviews, it was found that patients with confirmed previous treatments responded positively to participating in the study and reported that their symptoms improved from the baseline to week 12. This is normally a difficult treatment group, and little or no effect of other products is often reported. In future work, a comparative study should be carried out to establish whether a significant positive effect can be achieved compared with one or more of the treatment alternatives.

\section{Conclusion}

Promising results were found when women receiving adjuvant aromatase-inhibitor therapy and experiencing VVA symptoms were treated for 12 weeks with the water-based cellulose gel, a vaginal gel based on aqueous hydromellose. The gel treatment reduced the severity of the MBSs of VVA, slightly lowered vaginal $\mathrm{pH}$, reduced symptoms like vaginal dryness, dysuria, and dyspareunia, and led to improved QoL for the patients. No concerns relating to safety or tolerability were identified. In light of the benefits of using a nonhormone medication for treatment of VVA, and taking into account the highly significant positive efficacy found for the gel in this study, it can be considered a suitable candidate for first-choice treatment, especially among breast cancer 
patients who normally should not be treated with local estrogen ${ }^{19}$ and women predisposed to breast cancer.

\section{Abbreviations}

MBS, most bothersome symptom; VVA, vulvovaginal atrophy.; .

\section{Ethics Approval and Informed Consent}

This study was conducted in compliance with the International Conference on Harmonisation Harmonised Tripartite Guideline for good clinical practice guidelines and in accordance with ethical principles in the Declaration of Helsinki. The protocol was reviewed and approved by the Independent Ethics Committee (IEC) in Stockholm, Sweden (2019-02346), and all participants provided written informed consent.

\section{Acknowledgments}

Medical writing assistance for this publication was provided by Katarina Ortfelt, MSc, QARA Consulting AB.

\section{Funding}

This work was funded by Peptonic Medical AB, Sweden.

\section{Disclosure}

The authors report no conflicts of interest in this work.

\section{References}

1. Lethaby A, Ayeleke RO, Roberts H. Local oestrogen for vaginal atrophy in postmenopausal women. Cochrane Database Syst Rev. 2016;2016:Cd001500. doi:10.1002/14651858.CD001500

2. Iosif CS, Bekassy Z. Prevalence of genito-urinary symptoms in the late menopause. Acta Obstet Gynecol Scand. 1984;63(3):257-260. doi:10.3109/00016348409155509

3. Henriksson L, Stjernquist M, Boquist L, Cedergren I, Selinus I. A one-year multicenter study of efficacy and safety of a continuous, low-dose, estradiol-releasing vaginal ring (Estring) in postmenopausal women with symptoms and signs of urogenital aging. Am J Obstet Gynecol. 1996;174(1):85-92. doi:10.1016/s0002-9378(96)70378-2

4. Caillouette JC, Sharp CF Jr, Zimmerman GJ, Roy S. Vaginal pH as a marker for bacterial pathogens and menopausal status. Am J Obstet Gynecol. 1997;176(6):1270-1275;discussion 1275-1277. doi:10.1016/ s0002-9378(97)70345-4

5. Bachmann GA. The clinical platform for the 17beta-estradiol vaginal releasing ring. Am J Obstet Gynecol. 1998;178(5):S257-S260. doi:10.1016/s0002-9378(98)70558-76

6. Grady D. Clinical practice. Management of menopausal symptoms. N Engl J Med. 2006;355(22):2338-2347. doi:10.1056/NEJMcp054015

7. Cano A, Estévez J, Usandizaga R, et al. The therapeutic effect of a new ultra low concentration estriol gel formulation $(0.005 \%$ estriol vaginal gel) on symptoms and signs of postmenopausal vaginal atrophy: results from a pivotal Phase III Study. Menopause. 2012;19 (10):1130-1139. doi:10.1097/gme.0b013e3182518e9a
8. Freedman M, Kaunitz AM, Reape KZ, Hait H, Shu H. Twice-weekly synthetic conjugated estrogens vaginal cream for the treatment of vaginal atrophy. Menopause. 2009;16(4):735-741. doi:10.1097/ gme.0b013e318199e734

9. Fernandes T, Costa-Paiva LH, Pedro AO, Baccaro LFC, Pinto-Neto AM. Efficacy of vaginally applied estrogen, testosterone, or polyacrylic acid on vaginal atrophy: a randomized controlled trial. Menopause. 2016;23(7):792-798. doi:10.1097/gme.0000 000000000613

10. Society NAM. Management of symptomatic vulvovaginal atrophy: 2013 position statement of The North American Menopause Society. Menopause. 2013;20(9):888-902;quiz 903-884. doi:10.1097/ GME.0b013e3182a122c2

11. 1177 Vårdguiden. Klimakteriebesvär [Health care guide. Menopausal symptoms]; 2017. Available from: https://www.1177.se/Fakta-och$\mathrm{rad} /$ Sjukdomar/Klimakteriebesvar1/. Accessed January 12, 2018.

12. Ayehunie S, Wang YY, Landry T, Bogojevic S, Cone RA. Hyperosmolal vaginal lubricants markedly reduce epithelial barrier properties in a three-dimensional vaginal epithelium model. Toxicol Rep. 2018;5:134-140. doi:10.1016/j.toxrep.2017.12.011

13. Edwards D, Panay N. Treating vulvovaginal atrophy/genitourinary syndrome of menopause: how important is vaginal lubricant and moisturizer composition? Climacteric. 2016;19(2):151-161. doi:10.3109/13697137.2015.1124259

14. Lima SM, Bernardo BFA, Yamada SS, et al. Effects of Glycine max (L.) Merr. soy isoflavone vaginal gel on epithelium morphology and estrogen receptor expression in postmenopausal women: a 12-week, randomized, double-blind, placebo-controlled trial. Maturitas. 2014;78(3):205-211. doi:10.1016/j.maturitas.2014.04.007

15. Jokar A, Davari T, Asadi N, Ahmadi F, Foruhari S. Comparison of the hyaluronic acid vaginal cream and conjugated estrogen used in treatment of vaginal atrophy of menopause women: a randomized controlled clinical trial. Int $J$ Community Based Nurs Midwifery. 2016;4(1):69-78.

16. Lima SMRR, Yamada SS, Reis BF, et al. Effective treatment of vaginal atrophy with isoflavone vaginal gel. Maturitas. 2013;74 (3):252-258. doi:10.1016/j.maturitas.2012.11.012

17. Vagifem. Vagifem ${ }^{\circledR}$ (estradiol vaginal inserts) $10 \mathrm{mcg} ; 2017$. Available from: https://www.vagifem.com/. Accessed July 18, 2017.

18. Fianu Jonasson A, Bixo M, Sundström Poromaa I, Åstrom M. Safety and efficacy of an oxytocin gel and an equivalent gel but without hormonal ingredients (Vagivital ${ }^{\circledR} \mathrm{Gel}$ ) in postmenopausal women with symptoms of vulvovaginal atrophy: a Randomized, Double-Blind Controlled Study. Med Devices (Auckl). 2020;13:339-347. PMID: 33116956; PMCID: PMC7547122. doi:10.2147/MDER.S265824

19. Kendall A, Dowsett M, Folkered E, Smith I. Caution: vaginal estradiol appears to be contraindicated in postmenopaul women on adjuvant aromatase inhibitors. Ann Oncol. 2006;17(4):584-587. doi:10.1093/annonc/mdj127

20. Wysocki S, Kingsberg S, Krychman M. Management of vaginal atrophy - Implications REVIVE Study. Clin Med Insights Reprod Health. 2014. doi:10.4137/CMRH.S14498

21. Cagnacci A, Venier M, Xholli A, Paglietti C, Caruso S. Female sexuality and vaginal health across the menopausal age - NAMS. Menopause. 2019;27(1):14-19. doi:10.1097/GME.00000 00000001427

22. Simon JA, Kokot-Kierepa M, Goldstein J, Nappi RE. Vaginal health in the United States results from vaginal health insights. Menopause. 2013;20(10):1043/1048. doi:10.1097/gme.0b013e318287342d

23. Palacios S, Nappi RE, Bruyniks N, Particco M, Panay N. The European vulvovaginal epidemiological survey EVES prevalence symptoms and impact of vulvovaginal atrophy of menopause 2018. Climacteric. 2018;21:3. 


\section{Publish your work in this journal}

Medical Devices: Evidence and Research is an international, peerreviewed, open access journal that focuses on the evidence, technology, research, and expert opinion supporting the use and application of medical devices in the diagnosis, monitoring, treatment and management of clinical conditions and physiological processes. The identification of novel devices and optimal use of existing devices

which will lead to improved clinical outcomes and more effective patient management and safety is a key feature of the journal The manuscript management system is completely online and includes a very quick and fair peer-review system. Visit http:// www.dovepress.com/testimonials.php to read real quotes from published authors.

Submit your manuscript here: https://www.dovepress.com/medical-devices-evidence-and-research-journal 